\title{
Epigenetic Modification Drives Acute Kidney Injury-to-Chronic Kidney Disease Progression
}

\author{
Zhenzhen $\mathrm{Li}^{\mathrm{a}}$ Ningning $\mathrm{Li}^{\mathrm{b}}$ \\ aMedical Research Center, The First Affiliated Hospital of Zhengzhou University, Zhengzhou, PR China; \\ ${ }^{b}$ Department of Pathology, Henan Medical College, Zhengzhou, PR China
}

\section{Keywords}

Acute kidney injury · Chronic kidney disease - Epigenetic modifications $\cdot$ Histone modifications $\cdot$ DNA methylation

\begin{abstract}
Acute kidney injury (AKI) is a common clinical critical disease. Due to its high morbidity, increasing risk of complications, high mortality rate, and high medical costs, it has become a global concern for human health problems. Initially, researchers believed that kidneys have a strong ability to regenerate and repair, but studies over the past 20 years have found that kidneys damaged by AKI are often incomplete or even unable to repair. Even when serum creatinine returns to baseline levels, renal structural damage persists for a long time, leading to the development of chronic kidney disease (CKD). The mechanism of AKI-to-CKD transition has not been fully elucidated. As an important regulator of gene expression, epigenetic modifications, such as histone modification, DNA methylation, and noncoding RNAs, may play an important role in this process. Alterations in epigenetic modification are induced by hypoxia, thus promoting the expression of inflammatory factor-related genes and collagen secretion. This review elaborated the role of epigenetic modifications in AKI-to-CKD progression, the diagnostic value of epigenetic modifications biomarkers in AKI chronic outcome,
\end{abstract}

and the potential role of targeting epigenetic modifications in the prevention and treatment of AKI to CKD, in order to provide ideas for the subsequent establishment of targeted therapeutic strategies to prevent the progression of renal tubular-interstitial fibrosis.

(C) 2021 S. Karger AG, Base

\section{Introduction}

Acute kidney injury (AKI) is a global public health problem with high morbidity, high mortality, and high medical cost [1]. Globally, about 13.3 million people suffer from AKI each year, resulting in up to 2 million deaths $[2,3]$. In the past, AKI was thought to be a reversible disease but recent studies show that $20-50 \%$ of AKI survivors will progress to chronic kidney disease (CKD) [4]. Patients with AKI may develop CKD, even end-stage kidney disease, even if there is no history of kidney disease, or renal function has partially or fully recovered [4-9]. Compared with those without AKI, patients with AKI had an approximately 8 -fold increased risk of developing CKD and 3-fold of developing end-stage kidney disease [10]. Our previous study also showed that after ureteral obstruction causing AKI, mice developed pathological changes of CKD, such as renal fibrosis $[11,12]$. AKI can karger@karger.com www.karger.com/nef

(C) 2021 S. Karger AG, Base

Karger!
Correspondence to:

Zhenzhen Li, lzzkiki@126.com 
be caused by ischemia reperfusion, nephrotoxicity, and urinary tract obstruction. The mechanism involving AKI-to-CKD transition is not fully understood and may be associated with epigenetic modification, hypoxia, chronic inflammation, cell cycle arrest, mitochondrial dysfunction, senescence, and activation of the renin angiotensin system [13-17]. Severe tissue damage caused by AKI eventually leads to renal hypoxia, subsequent nonbacterial inflammation, and maladaptive repair. In other words, although there are many causes of AKI, the transition from AKI to CKD is mainly induced by hypoxia due to prior injury $[13,14,16]$. Hypoxia is a prerequisite and common pathway for epigenetic modification, chronic inflammation, apoptosis, and other mechanisms $[18,19]$. Hypoxia-initiated epigenetic modification changes are also known as hypoxia memory [19]. In the past few decades, a large number of studies have confirmed that epigenetic modifications (such as DNA methylation and histone modifications) play an important role in the occurrence and development of kidney disease and as an important target for the treatment of CKD have achieved good therapeutic effects in clinical practice. This article first briefly introduces epigenetics and the mechanisms of epigenetic changes in AKI-to-CKD transition, then focuses on the role of epigenetic modifications in the progression from $\mathrm{AKI}$ to $\mathrm{CKD}$, and finally reviews the value of epigenetic markers in the diagnosis of chronic regression of AKI and the potential value of targeted interventions for epigenetic modifications in the prevention and treatment of the transition from AKI to CKD.

\section{Epigenomics}

Epigenetics is a newly developed discipline in recent decades, which mainly studies the important role of reversible and heritable changes in gene function in regulating gene expression, trait inheritance, and disease occurrence in the absence of any changes in nuclear DNA sequence [20]. All cells in the body share the same DNA information but different organs express different genes, which is the result of epigenetic regulation. Specifically, epigenetic modification refers to heritable changes in gene activity independent of nucleotide sequence changes, including DNA methylation, histone modification, chromosomal remodeling, gene transcription, and translation activity changes mediated by RNA and RNA modification [21,22]. Current studies have found that epigenetic modification is significantly affected by changes of cell's internal and external environment and plays an im- portant regulatory role in the intergenerational inheritance of acquired traits, stem cell fate determination, and cancer development [23]. Changes in the epigenome may promote the progression of underlying diseases.

\section{The Mechanism of Epigenetic Changes in AKI-to-CKD Transition}

The most important pathological manifestation of AKI-to-CKD transition is hypoxia due to reduced peritubular capillaries and consequently apoptosis of renal tubular epithelial cells. Hypoxia plays a big part in AKI-toCKD progression and is the ultimate common pathway mediating AKI-to-CKD transition $[18,19]$. In the case of minor or short duration of renal injury, adaptive repair is necessary for renal function recovery, for example, the proliferation of surviving renal tubular epithelial cells instead of apoptotic epithelial cells. However, when the injury is severe or persistent, it leads to maladaptive repair, that is, aseptic inflammation and fibrosis [14]. After kidney injury, hypoxia induces renal inflammation and fibrosis, which leads to reduced peritubular capillaries, decreased oxygen diffusion efficiency, aggravated hypoxia, increased apoptosis of renal tubular epithelial cells, and finally the occurrence of CKD [24].

Renal tubular epithelial cells quickly expressed hypoxia inducible factor 1 (HIF-1) 3-7 days after renal ischemiareperfusion injury to mediate subsequent epigenetic modifications [25]. HIF- 1 consists of $\alpha$ - and $\beta$-units that bind to hypoxic response elements in the regulatory region of target genes to regulate their expression under hypoxia conditions. Epigenetic changes caused by hypoxia are stored in cells and have long-term effect, known as "hypoxia memory." These changes, including DNA methylation, histone modification, chromatin remodeling, and alterations in the level of noncoding RNA, can induce AKI-to-CKD transition long after the first full recovery from AKI. Epigenetic changes induced by hypoxia promote the expression of inflammatory and fibrotic genes such as monocyte chemoattractant protein 1 (MCP-1), transforming growth factor $\beta 1$ (TGF- $\beta 1$ ), and type III collagen $[19,25]$.

\section{The Role and Mechanism of Epigenetic Modification in AKI-to-CKD Transition}

\section{Histone Modification and Chromatin Remodeling}

The main chemical components of chromatin are DNA and histones. Histones are proteins rich in arginine 
and lysine, which contain 5 components: H1, H2A, H2B, $\mathrm{H} 3$, and $\mathrm{H} 4$. A variety of post-translational covalent modifications can occur on the amino terminus of histones, mainly including methylation, acetylation, phosphorylation, ubiquitination, SUMO, and so on [26]. Acetylation and deacetylation are catalyzed by histone acetyltransferase (HAT) and histone deacetylase (HDAC), respectively [22]. Histone modifications and chromatin remodeling are primarily associated with inflammatory and fibrotic gene expression, both of which can drive the activation of these genes [27]. Chromatin remodeling mostly relies on histone changes to achieve its effects, so the 2 will be discussed together.

\section{Histone Methylation}

Currently, there is no analysis of overall histone methylation in the field of kidney disease, and only a few specific histone methylations have been detected. Both histone 3 lysine 27 trimethylation and histone 3 lysine 4 trimethylation were significantly upregulated in unilateral ureteral obstruction (UUO) fibrotic kidneys and human CKD kidneys, which were closely associated with the fibrotic process $[28,29]$. After the onset of ischemia-reperfusion injury, increased $\mathrm{H} 3 \mathrm{~K} 4 \mathrm{me} 3$ methylation was closely associated with increased expression of inflammation-related genes (TNF- $\alpha$ ), fibrosis-related genes (TGF- $\beta 1$, type III collagen), and cholesterol-regulated genes (HMGRC), ultimately leading to a gradual transition from AKI to CKD [30-33]. The methylation process of the above histones is catalyzed by their specific histone methyltransferases, EZH2 (for H3K29me and H3K27me), and SET7/9 (for H3K4me), respectively. After AKI occurs, they induce their respective specific histone methylation and promote renal fibrosis. Renal inflammation or fibrosis was significantly improved after blockade, respectively, by specific inhibitors $3-\mathrm{DZNeP}(\mathrm{EZH} 2)$ and sinefungin (SET7/9) [34-37]. Lysine-specific demethylase KDM3A is induced by HIF-1 under hypoxic conditions and recruited at the glucose transporter 3 site to demethylate histone 3 lysine 9 dimethylated, maximizing the hypoxia-inducing effect of glucose transporter 3 and participating in the formation of "hypoxic memory" [38]. The above information can be referred to Tables 1 and 2 .

Histone Acetylation

Histone acetylation has been extensively studied in AKI and renal fibrosis. After ischemia injury, the histone acetylation level decreased overall and began to recover $24 \mathrm{~h}$ after reperfusion, which was related to decreased HAT activity during hypoxia and downregulation of
HDAC after reperfusion [39]. However, when renal fibrosis progressed after reperfusion, the level of $\mathrm{H} 3$ acetylation was much higher than the baseline value, accompanied by increased expression of inflammatory and fibrosis genes [39]. After 3 weeks of ischemia-reperfusion injury, acetylation of lysine residue 9 of histone 3 enhanced significantly, and expression of MCP-1, TGF- $\beta 1$, and collagen increased [40]. Acetylation of lysine residue 9 of histone 3 and $\mathrm{H} 3 \mathrm{~K} 4 \mathrm{Me} 3$ together induce the production and activation of cholesterol and activating transcription factor 3, the latter 2 cooperate to protect the kidney and alleviate renal injury to a certain extent [57].

As catalytic acetylation and deacetylation enzymes, HAT and HDAC act on their specific histones and play an important role in AKI-to-CKD transition [58]. Due to a large molecular size and easy detection, they are relatively easy to intervene in clinical practice and are expected to become a therapeutic target. The HAT inhibitor curcumin attenuates renal oxidative stress and promotes recovery of renal function, suggesting that HAT promotes renal injury to some extent [59]. HDAC is classified into 4 categories (I, II, III, and IV) according to its distribution and action, mainly inducing renal inflammation and fibrosis [58]. On the other hand, it also inhibits activating transcription factor 3 activity, causes chromatin agglutination, and to some extent inhibits the transcription of inflammatory genes such as IL-6 and Pselectin but that extent is not sufficient to reverse the damage [60]. HDAC inhibitors such as trichostatin A (TSA), phenylthiobutanoic acids (PTBAs) and PTBA analogs, and UPHD 25 and 186, have been reported to promote renal function recovery and reduce fibrosis after AKI but the mechanism of action is not exactly the same. TSA reduces platelet-derived growth factor-induced fibroblast proliferation after ischemia-reperfusion injury [41]. PTBA, as a small-molecule HDAC inhibitor, can promote renal recovery after AKI in zebrafish and mice, induce proliferation of renal tubular epithelial cells and reduce renal fibrosis [42]. As PTBA analogs, UPHD 25 inhibits the transition from AKI to CKD by increasing the expression of renal progenitor cell genes, enhancing renal tubular epithelial cell dedifferentiation, decreasing kidney injury molecule 1 expression, and reducing the number of infiltrating macrophages [43]. FR276457, as a pan-HDAC inhibitor, protects against UUO-induced renal interstitial fibrosis by preventing MCP-1 secretion [44]. Valproic acid, a class I HDAC inhibitor prevents the transition from AKI to CKD by reducing inflammatory cellular infiltration and proinflammatory cytokines [45]. The recent 
Table 1. Histone modification in AKI-to-CKD transition

\begin{tabular}{|c|c|c|c|c|}
\hline Histone modification & Model & Change in kidney injury & Effect on kidney & Ref. \\
\hline \multicolumn{5}{|l|}{ Histone methylation } \\
\hline H3K9me3 & UUO & Increased & Fibrosis $\uparrow$, inflammation $\uparrow$ & {$[28,29]$} \\
\hline H3K27me3 & CKD (humans) & Increased & Fibrosis $\uparrow$ & \\
\hline $\mathrm{H} 3 \mathrm{~K} 4 \mathrm{me} 3$ & IRI & Increased & Fibrosis $\uparrow$, inflammation $\uparrow$ & [30-33] \\
\hline \multicolumn{5}{|l|}{ Histone acetylation } \\
\hline $\mathrm{H} 3 \mathrm{~K} 9 \mathrm{Ac}$ & IRI & Transient $(<24 \mathrm{~h})$ decreased, then increased up to 3 weeks & Fibrosis $\uparrow$, inflammation $\uparrow$ & {$[40]$} \\
\hline
\end{tabular}

AKI, acute kidney injury; CKD, chronic kidney disease; UUO, unilateral ureteral obstruction; IRI, ischemia-reperfusion injury; H3K27me3, histone 3 lysine 27 trimethylation; H3K9me3, histone 3 lysine 4 trimethylation; H3Ac, H3 acetylation; H3K9Ac, acetylation of lysine residue 9 of histone 3.

Table 2. Targeted intervention for epigenetic modifications (histone modification and DNA methylation) in AKI-to-CKD transition

\begin{tabular}{|c|c|c|c|c|}
\hline Intervention & Model & Target & Effect on kidney & Ref. \\
\hline \multicolumn{5}{|l|}{ Histone methylation } \\
\hline 3-DZNeP & IRI & EZH2 & Fibrosis $\downarrow$, inflammation $\downarrow$ & {$[34]$} \\
\hline Sinefungin & UUO & SET7/9 & Fibrosis $\downarrow$, inflammation $\downarrow$ & {$[36]$} \\
\hline \multicolumn{5}{|l|}{ Histone acetylation } \\
\hline TSA & IRI & Class I and II HDAC inhibitor & Fibrosis $\downarrow$ & {$[41]$} \\
\hline PTBA & $\mathrm{AA}$ & Class I HDAC inhibitor & Fibrosis $\downarrow$, inflammation $\downarrow$ & {$[42]$} \\
\hline FR276457 & UUO & Class I and II HDAC inhibitor & Fibrosis $\downarrow$ & {$[44]$} \\
\hline Valproic acid & IRI & Class I HDAC inhibitor & Inflammation $\downarrow$, fibrosis $\downarrow$ & {$[45]$} \\
\hline \multirow[t]{4}{*}{ MS-275 } & LPS & Class I HDAC inhibitor & Inflammation $\downarrow$, renal function $\uparrow$ & {$[46]$} \\
\hline & UUO & & Fibrosis $\downarrow$ & {$[47]$} \\
\hline & IRI & & Fibrosis $\downarrow$ & {$[41]$} \\
\hline & Folic acid & & Renal injury $\uparrow$, generation $\downarrow$ & {$[48]$} \\
\hline \multicolumn{5}{|l|}{ DNA methylation } \\
\hline 5-aza-2'-deoxycytidine & UUO & DNMT inhibitor & Klotho $\uparrow$, fibrosis $\downarrow$ & {$[53]$} \\
\hline \multirow[t]{2}{*}{ 5-Azacytidine } & Cisplatin & DNMT inhibitor & Nephrotoxicity $\downarrow$ & [54] \\
\hline & UUO & & EPO $\uparrow$, anemia $\downarrow$ & {$[55]$} \\
\hline Hydralazine & IRI & Demethylating activity: induction of TET3 & Fibrosis $\downarrow$, renal function $\uparrow$ & {$[56]$} \\
\hline
\end{tabular}

AKI, acute kidney injury; CKD, chronic kidney disease; UUO, unilateral ureteral obstruction; TSA, trichostatin A; PTBA, phenylthiobutanoic acid; IRI, ischemia-reperfusion injury; AA, aristolochic acid; LPS, lipopolysaccharide; 3-DZNeP, 3-deazaneplanocin A; EPO, erythropoietin; DNMT, DNA methyltransferase; HDAC, histone deacetylase.

published article showed that HDAC inhibitor MS-275 pretreatment improved renal function and ameliorated renal inflammation and reactive oxygen species production in LPS-induced AKI mice [46]. Administration of MS-275 inhibited all UUO-induced fibrotic responses and suppressed TGF- $\beta 1$ production [47]. The same effect of MS-275 was also observed in the ischemia-reper- fusion injury model [41]. Interestingly, MS-275 did result in more severe tubular injury and failed tubular regeneration in the mouse model of AKI induced by folic acid, which was associated with activation of epidermal growth factor receptor signaling [48]. TMP195 and MC1568, selective class IIa HDAC inhibitors, were reported to have a powerful renoprotective effect in LPS 
and UUO-induced AKI mouse model, respectively [49, 50]. An HDAC3-selective inhibitor RGFP966 derepressed Klotho and mitigated the renal fibrotic injuries in both UUO and aristolochic acid nephropathy mice [51]. Ricolinostat (ACY-1215) was found to mitigate renal fibrosis in UUO-induced kidney injury [52]. The above information can be referred to Tables 1 and 2 .

\section{Other Modifications}

Other modifications may also be involved in the fibrosis process after AKI. Increased expression of histone $2 \mathrm{~A}$ variants coincided with increased expression of inflammatory and fibrotic genes [32]. The chromatin remodeling complex SWI/SNF, catalyzed by chromatin remodeling enzymes (such as brahma-related gene 1), causes chromatin agglutination, increased promoter accessibility, and upregulation of transcription of the above genes [61]. Endothelin-1 has a strong vasoconstriction effect and can aggravate renal hypoxia. Increased endothelin-1 after ischemia/ reperfusion injury was related to histone modification, but the specific mechanism remains unknown [62]. The above information can be referred to Tables 1 and 2 .

\section{DNA Methylation/Demethylation}

DNA methylation occurs mainly in the cytosine of CpG islands, where the fifth carbon atom of cytosine is methylated by DNA methyltransferases to form 5-methylcytosine [63]. DNA methylation is one of the most specifically characterized epigenetic modifications that can directly prevent transcription factors from binding to promoters and cause gene silencing. DNA methylation can be demethylated by demethyltransferase or oxidized from 5-methylcytosine to 5-hydroxymethylcytosine by 10/11 translocation protein [64].

Clinical evidence implies that hypermethylation of genes such as Klotho, erythropoietin, and Ras GTPaseactivating-like protein 1 (RASAL1) is involved in the pathogenesis of CKD after AKI and may play a role in promoting fibrosis $[55,65,66]$. DNMTS inhibitors such as 5-azacytidine, 5-aza-2-deoxycytidine, and the antihypertensive hydralazine retard the transition from AKI to CKD by reducing the methylation level of the above genes and upregulating their expression [55, 56, 67]. Methylation of the RASAL1 gene promotes the development of CKD after AKI. This gene inhibits fibroblast activity, but its expression is downregulated after methylation, which can activate fibroblasts, leading to increased secretion of TGF- $\beta$ and type I collagen and aggravated interstitial fibrosis. Compared with mice overexpressing RASAL1, fibrosis was more severe in mice with RASAL1 deletion

Epigenetic Modification Drives AKI

Progression
[68]. Demethylation treatment can effectively restore its expression that delays the transition from AKI to CKD. Methylation of RASAL1 mainly mediates the development of fibrosis after ischemia-reperfusion injury. Methylation of RASAL1 was not observed in mice with mild and reversible injury, whereas the level of RASAL1 methylation was positively correlated with renal interstitial fibrosis in mice with severe and irreversible injury. Low dose of hydralazine inhibits fibroblast activity by inducing RASAL1 demethylation, thereby inhibiting renal fibrosis after ischemia/reperfusion injury, and is expected to be a therapeutic tool to inhibit AKI-to-CKD transition [56].

Toxic kidney damage is also an important factor in the development of AKI and the progression of AKI to CKD. In mice with cisplatin-induced kidney injury, Tikoo et al. [54] found that the combination of 5-azacytidine with cisplatin led to remarkable reduction in nephrotoxicity by involving inhibition of cisplatin-induced metallothionein expression. Chang et al. [55] demonstrated that nontoxic doses of 5-azacytidine restored erythropoietin production and ameliorated anemia in the setting of renal fibrosis in UUO mice. Yin et al. [53] showed that 5-aza2-deoxycytidine abolished UUO-induced Klotho suppression and the promoter hypermethylation, thus attenuated pro-fibrotic protein expression and renal fibrosis. The above information can be referred to Table 2 .

\section{Noncoding RNAs}

Noncoding RNAs, mainly microRNA (miR) and long noncoding RNA (lncRNA), also play a role in kidney fibrosis (Fig. 1) [69]. LncRNAs, over 200 nucleotides in length, act as indicators or "signals" for transcriptional activity and participate in the regulation of downstream gene expression [70]. MiRs are short RNA molecules about 21-23 nucleotides long that regulate the expression of other genes and inhibit post-transcriptional gene expression by binding to the target mRNA [71].

LncRNAs play momentous roles in regulating different physiological processes such as cell proliferation, apoptosis, cell cycle progression, differentiation, and inflammation. The expression and role of IncRNAs in the transition from AKI to CKD are different. For example, nuclear enriched abundant transcript 1 (NEAT1), psoriasis susceptibility-related RNA gene induced by stress (PRINS), and LINC00520 mainly play a role in promoting the transition from $\mathrm{AKI}$ to CKD [72-74]. In contrast, aspartyl-tRNA synthetase antisense 1 (DARS-AS1), MALAT1, and lncRNA Miat inhibit this process $[70,75,76]$. After AKI occurred, NEAT1 and LINC00520 expressions were increased, both with miR-27 as a downstream gene, which promoted renal tubular epi- 


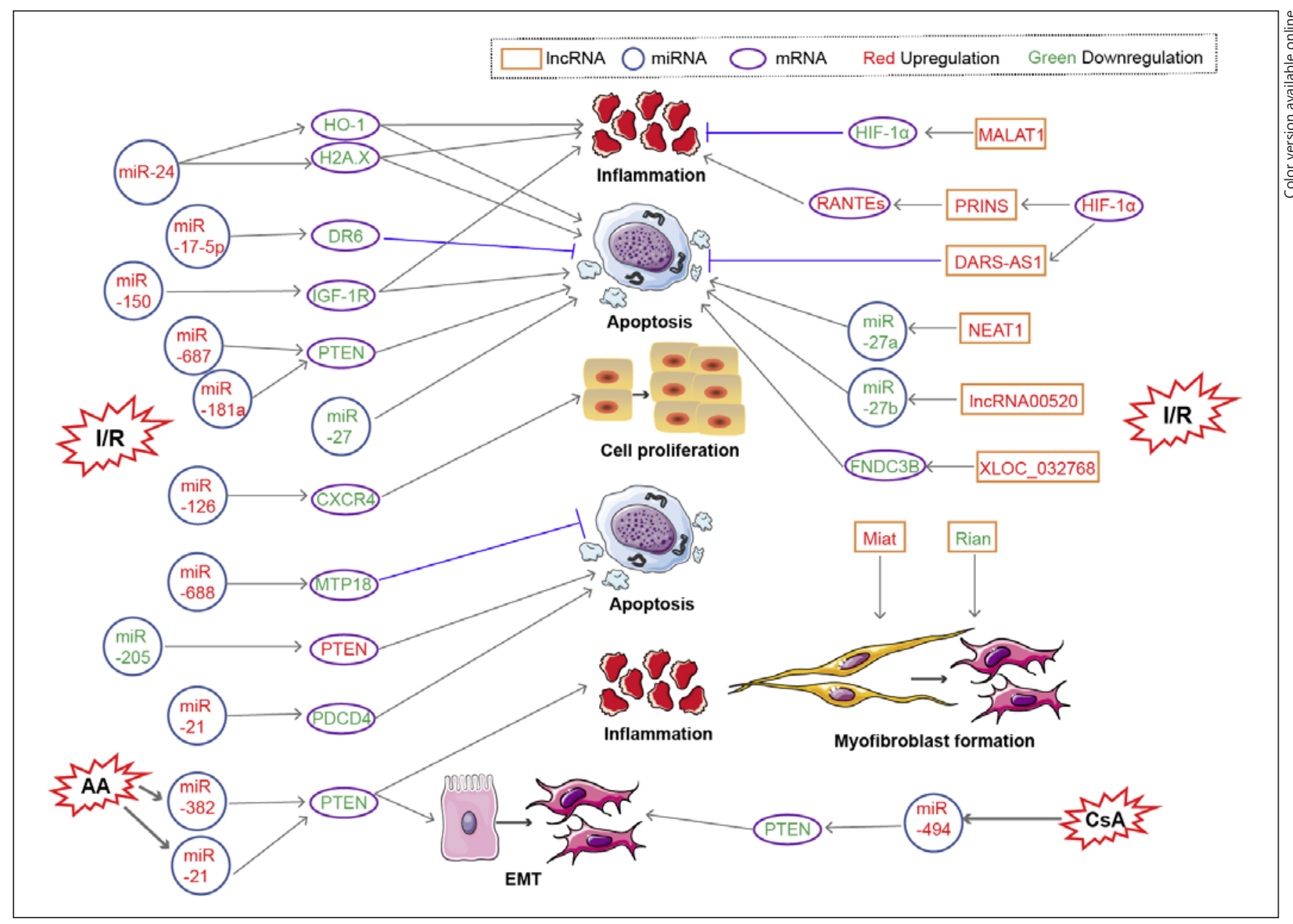

Fig. 1. Mechanism of noncoding RNAs involved in AKI-to-CKD transition. The mechanism of noncoding RNAs, mainly miR-and lncRNA-mediated renal fibrosis is summarized here. In I/R injury model, upregulation of miR-24, miR-17-5p, miR-150, miR-687, miR-181a, miR-126, miR-688, and miR-21, as well as downregulation of miR-27 and miR-205 activated corresponding downstream signaling pathways, and then affect these biological processes, including inflammation, apoptosis, and cell proliferation, ultimately leading to renal fibrosis. Upregulation of lncRNAs MALAT1, PRINS, DARS-AS1, NEAT1, IncRNA00520, XLOC_032768, and Miat, as well as downregulation of lncRNA Rian in I/R-induced AKI model activated corresponding downstream signaling pathways, which then affect various biological processes, including in-

thelial cell apoptosis and aggravated the renal injury of AKI by downregulating this $\mathrm{miR}[73,74]$. The results of flow cytometric analysis showed that NEAT1 expression was significantly higher in patients with ischemic AKI compared with normal controls. MALAT1 regulates the expression of HIF-1 $\alpha$, while PRINS and DARS-AS1 are induced by HIF-1 $\alpha$. MALAT1 acts on the NF- $\kappa B$ pathway to reduce flammation, apoptosis, and myofibroblast formation, ultimately leading to renal fibrosis. Upregulation of miR-382 and miR-21 in AA-induced AKI model, upregulation of miR-494 in CsA-induced AKI model activated corresponding downstream signaling pathways, which then affect various biological processes, including inflammation and tubular epithelial cell EMT, ultimately leading to renal fibrosis. AKI, acute kidney injury; $\mathrm{CKD}$, chronic kidney disease; miR, microRNA; lncRNA, long noncoding RNA; NEAT1, nuclear enriched abundant transcript 1; PRINS, psoriasis susceptibility-related RNA gene induced by stress; DARS-AS1, aspartyltRNA synthetase antisense 1; AA, aristolochic acid; EMT, epithelial-mesenchymal transition; CsA, cyclosporine A; I/R, ischemia/ reperfusion.
HIF-1 $\alpha$ expression and inhibit the subsequent inflammatory response. HIF-1a mediates the upregulation of both PRINS and DARS-AS1 but they have different mechanisms of action. DARS-AS1 inhibits renal tubular epithelial cell apoptosis, while PRINS is involved in the inflammatory response after AKI. The target gene of PRINS is RANTES, which is an inflammatory factor with strong chemotaxis ef- 
fect. PRINS upregulates the expression of RANTES and directly causes significant renal inflammatory response. Recently, Zhou et al. [77] identified lncRNA XLOC_032768 as a novel target in renal ischemia-reperfusion injury and its overexpression repressed the expression of fibronectin type III domain containing 3B and tubular epithelial cells apoptosis. Moreover, in the same mouse ischemia/reperfusion injury model, lncRNA Miat was upregulated and enhanced fibroblast formation, while lncRNA Rian was downregulated and also promoted fibroblast formation, both of which played different roles in the subsequent renal fibrosis of AKI, respectively. Thus, it can be seen that different lncRNAs are expressed in bidirection during the transition from AKI to CKD, and even if they are equally up or downregulated, they may play same roles.

miRs play an important role in kidney development, homeostasis, and disease and have been most studied in relation to disease. MiRs bind to target genes to regulate the inflammatory response, apoptosis, and cell cycle during the damage and repair stage after AKI, and play a corresponding role in the transition to CKD. For example, miR-27a-3p, miR-205, miR-17-5p, miR-126, and miR688 can inhibit the conversion of AKI to CKD by improving renal function, reducing fibrosis and inflammatory response $[73,78-81]$; on the contrary, miR-24, miR-494, miR-150, miR-181a, and miR-687 can promote this process [82-86]. miR-21 is a "double-edged sword," with mild upregulation inhibiting fibrosis and inflammatory response but sustained upregulation promoting tubulointerstitial fibrosis after AKI [87]. As a downstream gene of several lncRNAs, miR-27a-3p inhibits kidney injury and promotes renal recovery. miR-205 provides a protective effect by inhibiting proline hydroxylase 1 and reducing intracellular reactive oxygen species. Upregulation of miR-17-5p plays a role in preventing AKI deterioration by inhibiting apoptosis or inflammatory response. miR-126, on the other hand, promotes the recovery of ischemia/reperfusion injury kidney by enhancing the regeneration of peritubular capillaries. miR-688 is induced by HIF-1 and protects renal function by maintaining mitochondrial dynamics in renal tubular epithelial cells. Upregulated miR-687 and miR-181a promote apoptosis and kidney injury by inhibiting PTEN. Recently, upregulation of miR-382 was also demonstrated to contribute to renal fibrosis secondary to aristolochic acid-induced kidney injury via PTEN signaling pathway [88]. The upregulation of miR-24 expression can mediate the development of CKD by inducing apoptosis in capillary endothelial cells and renal tubular epithelial cells. Similarly, miR-494 (promoting tubular epithelial cell epithelial-mesenchy-

Epigenetic Modification Drives AKI

Progression mal transition) and miR-150 (inducing inflammatory response and mesenchymal apoptosis) have been reported to promote the conversion of AKI to CKD.

\section{Targeted Therapy for AKI-to-CKD Transition}

Inhibition of the transition from AKI to CKD is an important means to improve the prognosis of AKI. Epigenetic modification plays a key role in the pathogenesis of disease and its targeted intervention can directly block the occurrence or progression of disease. The use of targeted intervention drugs to treat $\mathrm{AKI}$ and inhibit the occurrence of CKD is one of the future trends. DNMT inhibitors such as 5-azacytidine, 5-aza-2'-deoxycytidine, and low-dose hydralazine could effectively inhibit the progression of renal interstitial fibrosis after renal ischemia-reperfusion injury $[55,56,67]$. The HDAC inhibitors, such as TSA, PTBA, and their analogs UPHD 25 and 186 have been reported to reduce renal injury, promote recovery of renal function, reduce fibrosis, and delay the transition from AKI to CKD in mouse AKI models [4143]. In the future, HDAC inhibitors may be considered to treat AKI and block chronic outcomes after AKI. Inhibition of histone methyltransferase EZH2 can also be a new target for improving renal fibrosis. The above-mentioned noncoding RNAs also begin to perform in promoting or inhibiting the transition from AKI to CKD. By upregulating noncoding RNAs such as DARS-AS1, MATALT1, miR-687, and miR-27a-3p, which inhibit AKI-to-CKD transition, or downregulating noncoding RNAs such as PRINS, NEAT1, miR-24, miR-494, and miR-150, which promote the development of fibrosis after AKI, it is expected to directly interfere with the disease progression epigenetically. In a word, targeted intervention of epigenetic factors is expected to be a new approach to target inhibit the transition from AKI to CKD and improve the prognosis of AKI but continuous efforts are needed to successfully introduce it into clinical use.

\section{Summary and Future Perspectives}

It is an undisputed fact that AKI tends to be chronic. Therefore, all AKI patients (regardless of partial or complete recovery of renal function) should be reviewed after discharge and the progression of AKI to CKD should be diagnosed and monitored in a timely manner so that appropriate interventions can be taken depending on the degree of progression. The main challenge is to obtain 


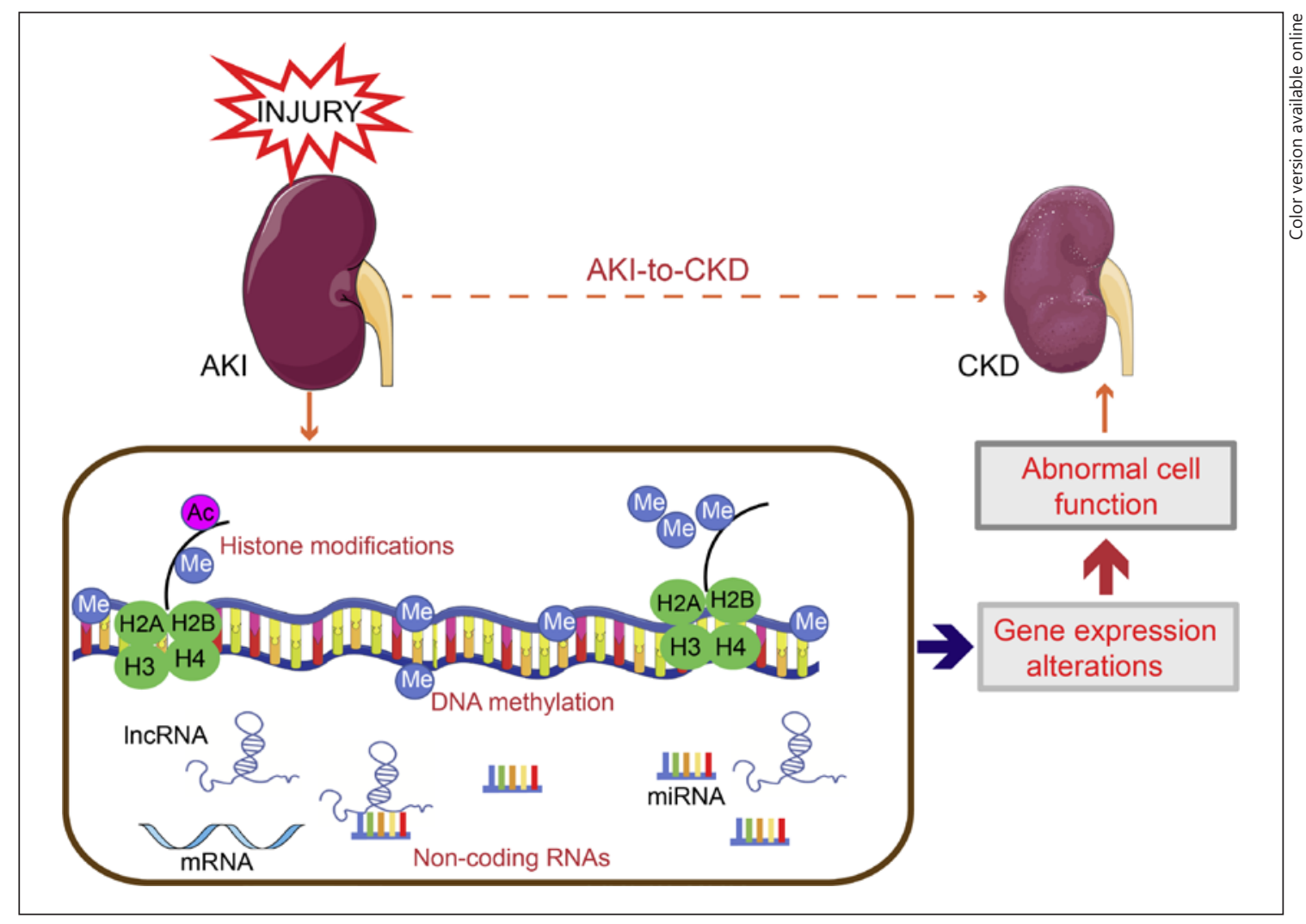

Fig. 2. Epigenetic modifications participate in the development of AKI-to-CKD transition. Increasing evidence points to the involvement of epigenetic regulation in the occurrence and progression of AKI to CKD. AKI can induce epigenetic changes including DNA methylation, histone modification, or changes in noncoding RNAs expression. The effect of epigenetic modifications on gene expression and cellular function plays a key role in the development of CKD. Me, methylation; Ac, acetylation; AKI, acute kidney injury; CKD, chronic kidney disease.

reliable biomarkers for clinical monitoring of disease changes during the transition from AKI to CKD. Epigenetics is pivotal in the occurrence and progression of AKI to CKD, as shown in Figure 2. Without altering the DNA sequence, epigenetics acts at the transcriptional and translational levels and then participates in various pathophysiological changes of AKI to CKD. In addition, epigenetic modification molecules are quite stable in both liquid and tissue specimens commonly used in clinical practice. Epigenetic modifications have been included in the field of biomarker detection due to their high stability, simple and mature detection technique, and a wide range of specimen sources (blood, urine, saliva, and tissue). Especially, when noncoding RNAs are included in exosomes, apoptotic bodies, and extracellular vesicles, it is important to recognize new noncoding RNAs as biomarkers. Compared with traditional histological examination of the biopsied tissue, the noninvasive diagnostic or prognostic noncoding RNAs can reduce patient's dis- comfort, the risk of complications, variability in histopathology interpretation, and high costs. Therefore, noncoding RNAs could potentially provide vital diagnostic and prognostic information and help to predict response to treatment in several clinical settings. Furthermore, epigenetics is reversible compared to genetic factors, making targeted interventions easier to achieve and promising as a treatment to delay the disease. Thus, besides explaining the mechanism of the occurrence and development of diseases, epigenetics is increasingly being applied at the clinical level. In summary, epigenetic modification, as one of the important modifications in the transition from $\mathrm{AKI}$ to $\mathrm{CKD}$, is of a great potential clinical value and research prospects both as a biomarker for predicting lesions and as a therapeutic tool for targeted intervention. In the future, more studies on epigenetics and related drug research and development are needed to provide reliable diagnostic markers and new therapeutic targets for the progression of AKI to CKD by changing epigenetics. 


\section{Statement of Ethics}

The study is exempt from Ethical Committee approval.

\section{Conflict of Interest Statement}

The authors have no conflicts of interest to declare.

\section{Funding Sources}

This work was supported by the Henan Province Medical Science and Technology Research Project (Grant No. SB201904001).

\section{Author Contributions}

Z.L. designed the paper, N.L. drafted the paper, and Z.L. revised the paper.

\section{References}

1 Büttner S, Stadler A, Mayer C, Patyna S, Betz $\mathrm{C}$, Senft $\mathrm{C}$, et al. Incidence, risk factors, and outcome of acute kidney injury in neurocritical care. J Intensive Care Med. 2020 Apr; 35(4):338-46.

2 Lewington AJ, Cerdá J, Mehta RL. Raising awareness of acute kidney injury: a global perspective of a silent killer. Kidney Int. 2013 Sep; 84(3):457-67.

3 Mehta RL, Cerdá J, Burdmann EA, Tonelli M, García-García G, Jha V, et al. International Society of Nephrology's 0by25 initiative for acute kidney injury (zero preventable deaths by 2025): a human rights case for nephrology. Lancet. 2015 Jun 27;385(9987):2616-43.

4 Kurzhagen JT, Dellepiane S, Cantaluppi V, Rabb H. AKI: an increasingly recognized risk factor for CKD development and progression. J Nephrol. 2020 Dec;33(6):1171-87.

5 Chawla LS, Bellomo R, Bihorac A, Goldstein SL, Siew ED, Bagshaw SM, et al. Acute kidney disease and renal recovery: consensus report of the acute disease quality initiative (ADQI) 16 workgroup. Nat Rev Nephrol. 2017 Apr; 13(4):241-57.

6 Hannan M, Ansari S, Meza N, Anderson AH, Srivastava A, Waikar S, et al. Risk factors for CKD progression: overview of findings from the CRIC study. Clin J Am Soc Nephrol. 2021; 16(4):648-59.

7 Jiang M, Bai M, Lei J, Xie Y, Xu S, Jia Z, et al. Mitochondrial dysfunction and the AKI to CKD transition. Am J Physiol Renal Physiol. 2020 Dec 1;319(6):F1105-16.

8 Li C, Shen Y, Huang L, Liu C, Wang J. Senolytic therapy ameliorates renal fibrosis postacute kidney injury by alleviating renal senescence. Faseb J. 2021 Jan;35(1):e21229.

9 Wen Y, Parikh CR. The aftermath of AKI: recurrent AKI, acute kidney disease, and CKD progression. J Am Soc Nephrol. 2021 Jan; 32(1):2-4.

10 Coca SG, Singanamala S, Parikh CR. Chronic kidney disease after acute kidney injury: a systematic review and meta-analysis. Kidney Int. 2012 Mar;81(5):442-8.

11 Li Z, Wang Y, Sun N, Liu X, Song E, Zhang Z, et al. Melatonin therapy protects against renal injury before and after release of bilateral ureteral obstruction in rats. Life Sci. 2019 Jul 15; 229:104-15.
12 Liu X, Sun N, Mo N, Lu S, Song E, Ren C, et al. Quercetin inhibits kidney fibrosis and the epithelial to mesenchymal transition of the renal tubular system involving suppression of the Sonic Hedgehog signaling pathway. Food Funct. 2019 Jun 19;10(6):3782-97.

13 Fiorentino M, Grandaliano G, Gesualdo L, Castellano G. Acute kidney injury to chronic kidney disease transition. Contrib Nephrol. 2018;193:45-54.

14 Guzzi F, Cirillo L, Roperto RM, Romagnani P, Lazzeri E. Molecular mechanisms of the acute kidney injury to chronic kidney disease transition: an updated view. Int J Mol Sci. 2019 Oct 6;20(19):4941.

15 Ogbadu J, Singh G, Aggarwal D. Factors affecting the transition of acute kidney injury to chronic kidney disease: potential mechanisms and future perspectives. Eur J Pharmacol. 2019 Dec 15;865:172711.

16 Ullah MM, Basile DP. Role of renal hypoxia in the progression from acute kidney injury to chronic kidney disease. Semin Nephrol. 2019 Nov;39(6):567-80.

17 do Valle Duraes F, Lafont A, Beibel M, Martin K, Darribat K, Cuttat R, et al. Immune cell landscaping reveals a protective role for regulatory $\mathrm{T}$ cells during kidney injury and fibrosis. JCI Insight. $2020 \mathrm{Feb}$ 13;5(3):e130651.

18 Tanaka S, Tanaka T, Nangaku M. Hypoxia as a key player in the AKI-to-CKD transition. Am J Physiol Renal Physiol. 2014 Dec 1; 307(11):F1187-95.

19 Tanaka T. Epigenetic changes mediating transition to chronic kidney disease: hypoxic memory. Acta Physiol. 2018 Apr;222(4): e13023.

20 Egger G, Liang G, Aparicio A, Jones PA. Epigenetics in human disease and prospects for epigenetic therapy. Nature. 2004 May 27; 429(6990):457-63.

21 Kelsey G, Stegle O, Reik W. Single-cell epigenomics: recording the past and predicting the future. Science. 2017 Oct 6;358(6359):6975.

22 Feinberg AP. The key role of epigenetics in human disease prevention and mitigation. $\mathrm{N}$ Engl J Med. 2018 Apr 5;378(14):1323-34.

23 Lyko F. The DNA methyltransferase family: a versatile toolkit for epigenetic regulation. Nat Rev Genet. 2018 Feb;19(2):81-92.
24 Guo C, Dong G, Liang X, Dong Z. Epigenetic regulation in AKI and kidney repair: mechanisms and therapeutic implications. Nat Rev Nephrol. 2019 Apr;15(4):220-39.

25 Shu S, Wang Y, Zheng M, Liu Z, Cai J, Tang $\mathrm{C}$, et al. Hypoxia and hypoxia-inducible factors in kidney injury and repair. Cells. 2019 Feb 28;8(3):207.

26 Zhang Y, Sun Z, Jia J, Du T, Zhang N, Tang Y, et al. Overview of histone modification. Adv Exp Med Biol. 2021;1283:1-16.

27 Portela A, Esteller M. Epigenetic modifications and human disease. Nat Biotechnol. 2010 Oct;28(10):1057-68.

28 Zhou X, Zang X, Ponnusamy M, Masucci MV, Tolbert E, Gong R, et al. Enhancer of zeste homolog 2 inhibition attenuates renal fibrosis by maintaining Smad7 and phosphatase and tensin homolog expression. J Am Soc Nephrol. 2016 Jul;27(7):2092-108.

29 Hewitson TD, Holt SG, Tan SJ, Wigg B, Samuel CS, Smith ER. Epigenetic modifications to $\mathrm{H} 3 \mathrm{~K} 9$ in renal tubulointerstitial cells after unilateral ureteric obstruction and TGF- $\beta 1$ stimulation. Front Pharmacol. 2017;8:307.

30 Naito M, Bomsztyk K, Zager RA. Endotoxin mediates recruitment of RNA polymerase II to target genes in acute renal failure. J Am Soc Nephrol. 2008 Jul;19(7):1321-30.

31 Naito M, Bomsztyk K, Zager RA. Renal ischemia-induced cholesterol loading: transcription factor recruitment and chromatin remodeling along the HMG CoA reductase gene. Am J Pathol. 2009 Jan;174(1):54-62.

32 Zager RA, Johnson AC. Renal ischemia-reperfusion injury upregulates histone-modifying enzyme systems and alters histone expression at proinflammatory/profibrotic genes. Am J Physiol Renal Physiol. 2009 May;296(5): F1032-41.

33 Johnson AC, Ware LB, Himmelfarb J, Zager RA. HMG-CoA reductase activation and urinary pellet cholesterol elevations in acute kidney injury. Clin J Am Soc Nephrol. 2011 Sep; 6(9):2108-13.

34 Sasaki K, Doi S, Nakashima A, Irifuku T, Yamada K, Kokoroishi K, et al. Inhibition of SET domain-containing lysine methyltransferase 7/9 ameliorates renal fibrosis. J Am Soc Nephrol. 2016 Jan;27(1):203-15.

35 Fontecha-Barriuso M, Martin-Sanchez D, 
Ruiz-Andres O, Poveda J, Sanchez-Niño MD, Valiño-Rivas L, et al. Targeting epigenetic DNA and histone modifications to treat kidney disease. Nephrol Dial Transplant. 2018 Nov 1;33(11):1875-86.

36 Liang H, Huang Q, Liao MJ, Xu F, Zhang T, $\mathrm{He}$, et al. EZH2 plays a crucial role in ischemia/reperfusion-induced acute kidney injury by regulating p38 signaling. Inflamm Res. 2019 Apr;68(4):325-36.

37 Yu C, Zhuang S. Histone methyltransferases as therapeutic targets for kidney diseases. Front Pharmacol. 2019;10:1393.

38 Mimura I, Nangaku M, Kanki Y, Tsutsumi S, Inoue T, Kohro T, et al. Dynamic change of chromatin conformation in response to hypoxia enhances the expression of GLUT3 (SL$\mathrm{C} 2 \mathrm{~A} 3$ ) by cooperative interaction of hypoxiainducible factor 1 and KDM3A. Mol Cell Biol. 2012 Aug;32(15):3018-32.

39 Marumo T, Hishikawa K, Yoshikawa M, Fujita T. Epigenetic regulation of BMP7 in the regenerative response to ischemia. J Am Soc Nephrol. 2008 Jul;19(7):1311-20.

40 Zager RA, Johnson AC, Becker K. Acute unilateral ischemic renal injury induces progressive renal inflammation, lipid accumulation, histone modification, and "end-stage" kidney disease. Am J Physiol Renal Physiol. 2011 Dec;301(6):F1334-45.

41 Levine MH, Wang Z, Bhatti TR, Wang Y, Aufhauser DD, McNeal S, et al. Class-specific histone/protein deacetylase inhibition protects against renal ischemia reperfusion injury and fibrosis formation. Am J Transplant. 2015 Apr;15(4):965-73.

42 Novitskaya T, McDermott L, Zhang KX, Chiba T, Paueksakon P, Hukriede NA, et al. A PTBA small molecule enhances recovery and reduces postinjury fibrosis after aristolochic acid-induced kidney injury. Am J Physiol Renal Physiol. 2014 Mar 1;306(5): F496-504.

43 Skrypnyk NI, Sanker S, Skvarca LB, Novitskaya T, Woods C, Chiba T, et al. Delayed treatment with PTBA analogs reduces postinjury renal fibrosis after kidney injury. Am J Physiol Renal Physiol. 2016 Apr 15;310(8): F705-16.

44 Kinugasa F, Noto T, Matsuoka H, Urano Y, Sudo Y, Takakura S, et al. Prevention of renal interstitial fibrosis via histone deacetylase inhibition in rats with unilateral ureteral obstruction. Transpl Immunol. 2010 May;23(12):18-23.

45 Costalonga EC, Silva FM, Noronha IL. Valproic acid prevents renal dysfunction and inflammation in the ischemia-reperfusion injury model. Biomed Res Int. 2016;2016: 5985903.

46 Zhang H, Zhang W, Jiao F, Li X, Zhang H, Wang $L$, et al. The nephroprotective effect of MS-275 on lipopolysaccharide (LPS)-induced acute kidney injury by inhibiting reactive oxygen species (ROS)-oxidative stress and endoplasmic reticulum stress. Med Sci Monit. 2018 Apr 28;24:2620-30.
47 Liu N, He S, Ma L, Ponnusamy M, Tang J, Tolbert E, et al. Blocking the class I histone deacetylase ameliorates renal fibrosis and inhibits renal fibroblast activation via modulating TGF-beta and EGFR signaling. PLoS One. 2013;8(1):e54001.

48 Tang J, Yan Y, Zhao TC, Gong R, Bayliss G, Yan $\mathrm{H}$, et al. Class I HDAC activity is required for renal protection and regeneration after acute kidney injury. Am J Physiol Renal Physiol. 2014 Aug 1;307(3):F303-16.

49 Xiong C, Guan Y, Zhou X, Liu L, Zhuang MA, Zhang W, et al. Selective inhibition of class IIa histone deacetylases alleviates renal fibrosis. Faseb J. 2019 Jul;33(7):8249-62.

50 Zhang W, Guan Y, Bayliss G, Zhuang S. Class IIa HDAC inhibitor TMP195 alleviates lipopolysaccharide-induced acute kidney injury. Am J Physiol Renal Physiol. 2020 Dec 1; 319(6):F1015-26.

51 Chen F, Gao Q, Wei A, Chen X, Shi Y, Wang $\mathrm{H}$, et al. Histone deacetylase 3 aberration inhibits Klotho transcription and promotes renal fibrosis. Cell Death Differ. 2021;28(3): 1001-12.

52 Chen X, Yu C, Hou X, Li J, Li T, Qiu A, et al. Histone deacetylase 6 inhibition mitigates renal fibrosis by suppressing TGF- $\beta$ and EGFR signaling pathways in obstructive nephropathy. Am J Physiol Renal Physiol. 2020 Dec 1; 319(6):F1003-14.

53 Yin S, Zhang Q, Yang J, Lin W, Li Y, Chen F, et al. TGF $\beta$-incurred epigenetic aberrations of miRNA and DNA methyltransferase suppress Klotho and potentiate renal fibrosis. Biochim Biophys Acta Mol Cell Res. 2017 Jul;1864(7): 1207-16.

54 Tikoo K, Ali IY, Gupta J, Gupta C. 5-Azacytidine prevents cisplatin induced nephrotoxicity and potentiates anticancer activity of cisplatin by involving inhibition of metallothionein, pAKT and DNMT1 expression in chemical induced cancer rats. Toxicol Lett. 2009 Dec 15;191(2-3):158-66.

55 Chang YT, Yang CC, Pan SY, Chou YH, Chang FC, Lai CF, et al. DNA methyltransferase inhibition restores erythropoietin production in fibrotic murine kidneys. J Clin Invest. $2016 \mathrm{Feb} ; 126(2): 721-31$.

56 Tampe B, Steinle U, Tampe D, Carstens JL, Korsten P, Zeisberg EM, et al. Low-dose hydralazine prevents fibrosis in a murine model of acute kidney injury-to-chronic kidney disease progression. Kidney Int. 2017 Jan;91(1):157-76.

57 Tang J, Zhuang S. Histone acetylation and DNA methylation in ischemia/reperfusion injury. Clin Sci. 2019 Feb 28;133(4):597-609.

58 Hyndman KA. Histone deacetylases in kidney physiology and acute kidney injury. Semin Nephrol. 2020 Mar;40(2):138-47.

59 Hassan FU, Rehman MS, Khan MS, Ali MA, Javed A, Nawaz A, et al. Curcumin as an alternative epigenetic modulator: mechanism of action and potential effects. Front Genet. 2019; 10:514.

60 Li HF, Cheng CF, Liao WJ, Lin H, Yang RB. ATF3-mediated epigenetic regulation pro- tects against acute kidney injury. J Am Soc Nephrol. 2010 Jun;21(6):1003-13.

61 Rodríguez-Romo R, Berman N, Gómez A, Bobadilla NA. Epigenetic regulation in the acute kidney injury to chronic kidney disease transition. Nephrology. 2015 Oct;20(10): 736-43.

62 Zager RA, Johnson AC, Andress D, Becker K. Progressive endothelin-1 gene activation initiates chronic/end-stage renal disease following experimental ischemic/reperfusion injury. Kidney Int. 2013 Oct;84(4):703-12.

63 Moore LD, Le T, Fan G. DNA methylation and its basic function. Neuropsychopharmacology. 2013 Jan;38(1):23-38.

64 Parry A, Rulands S, Reik W. Active turnover of DNA methylation during cell fate decisions. Nat Rev Genet. 2021 Jan;22(1):59-66.

65 Zhang C, Liang Y, Lei L, Zhu G, Chen X, Jin $T$, et al. Hypermethylations of RASAL1 and KLOTHO is associated with renal dysfunction in a Chinese population environmentally exposed to cadmium. Toxicol Appl Pharmacol. 2013 Aug 15;271(1):78-85.

66 Xu X, Tan X, Tampe B, Wilhelmi T, Hulshoff MS, Saito S, et al. High-fidelity CRISPR/Cas9based gene-specific hydroxymethylation rescues gene expression and attenuates renal fibrosis. Nat Commun. 2018 Aug 29;9(1):3509.

67 Chen J, Zhang X, Zhang H, Liu T, Zhang H, Teng J, et al. Indoxyl sulfate enhance the hypermethylation of Klotho and promote the process of vascular calcification in chronic kidney disease. Int J Biol Sci. 2016;12(10): 1236-46.

68 Bechtel W, McGoohan S, Zeisberg EM, Müller GA, Kalbacher H, Salant DJ, et al. Methylation determines fibroblast activation and fibrogenesis in the kidney. Nat Med. 2010 May;16(5):544-50.

69 Liu Z, Wang Y, Shu S, Cai J, Tang C, Dong Z. Non-coding RNAs in kidney injury and repair. Am J Physiol Cell Physiol. 2019 Aug 1; 317(2):C177-88.

70 Chen $\mathrm{H}$, Fan $\mathrm{Y}$, Jing $\mathrm{H}$, Tang S, Zhou J. Emerging role of lncRNAs in renal fibrosis. Arch Biochem Biophys. 2020 Oct 15;692: 108530.

71 Fan Y, Chen H, Huang Z, Zheng H, Zhou J. Emerging role of miRNAs in renal fibrosis. RNA Biol. 2020 Jan;17(1):1-12.

72 Yu TM, Palanisamy K, Sun KT, Day YJ, Shu $\mathrm{KH}$, Wang IK, et al. RANTES mediates kidney ischemia reperfusion injury through a possible role of HIF- $1 \alpha$ and LncRNA PRINS. Sci Rep. 2016 Jan 4;6:18424.

73 Jiang X, Li D, Shen W, Shen X, Liu Y. LncRNA NEAT1 promotes hypoxia-induced renal tubular epithelial apoptosis through downregulating miR-27a-3p. J Cell Biochem. 2019 Sep; 120(9):16273-82.

74 Tian X, Ji Y, Liang Y, Zhang J, Guan L, Wang C. LINC00520 targeting miR-27b-3p regulates OSMR expression level to promote acute kidney injury development through the PI3K/ AKT signaling pathway. J Cell Physiol. 2019 Aug;234(8):14221-33. 
75 Tian H, Wu M, Zhou P, Huang C, Ye C, Wang L. The long non-coding RNA MALAT1 is increased in renal ischemia-reperfusion injury and inhibits hypoxia-induced inflammation. Ren Fail. 2018 Nov;40(1):527-33.

76 Bijkerk R, Au YW, Stam W, Duijs JMGJ, Koudijs A, Lievers E, et al. Long non-coding RNAs rian and miat mediate myofibroblast formation in kidney fibrosis. Front Pharmacol. 2019;10:215.

77 Zhou X, Li Y, Wu C, Yu W, Cheng F. Novel lncRNA XLOC_032768 protects against renal tubular epithelial cells apoptosis in renal ischemia-reperfusion injury by regulating FNDC3B/TGF- $\beta 1$. Ren Fail. 2020 Nov;42(1):9941003.

78 Bijkerk R, van Solingen C, de Boer HC, van der Pol P, Khairoun M, de Bruin RG, et al. Hematopoietic microRNA-126 protects against renal ischemia/reperfusion injury by promoting vascular integrity. I Am Soc Nephrol. 2014 Aug;25(8):1710-22.

79 Hao J, Wei Q, Mei S, Li L, Su Y, Mei C, et al. Induction of microRNA-17-5p by $\mathrm{p} 53$ protects against renal ischemia-reperfusion injury by targeting death receptor 6 . Kidney Int. 2017 Jan;91(1):106-18.
80 Wei Q, Sun H, Song S, Liu Y, Liu P, Livingston MJ, et al. MicroRNA-668 represses MTP18 to preserve mitochondrial dynamics in ischemic acute kidney injury. J Clin Invest. 2018 Dec 3; 128(12):5448-64.

81 Chen W, Ruan Y, Zhao S, Ning J, Rao T, Yu W, et al. MicroRNA-205 inhibits the apoptosis of renal tubular epithelial cells via the PTEN/Akt pathway in renal ischemia-reperfusion injury. Am J Transl Res. 2019;11(12): 7364-75.

82 Lorenzen JM, Kaucsar T, Schauerte C, Schmitt R, Rong S, Hübner A, et al. MicroRNA-24 antagonism prevents renal ischemia reperfusion injury. J Am Soc Nephrol. 2014 Dec;25(12): 2717-29.

83 Bhatt K, Wei Q, Pabla N, Dong G, Mi QS, Liang $\mathrm{M}$, et al. MicroRNA-687 induced by hypoxia-inducible factor- 1 targets phosphatase and tensin homolog in renal ischemia-reperfusion injury. J Am Soc Nephrol. 2015 Jul; 26(7):1588-96.
84 Yuan J, Benway CJ, Bagley J, Iacomini J. MicroRNA-494 promotes cyclosporine-induced nephrotoxicity and epithelial to mesenchymal transition by inhibiting PTEN. Am J Transplant. 2015 Jun;15(6):1682-91.

85 Guan H, Peng R, Mao L, Fang F, Xu B, Chen M. Injured tubular epithelial cells activate fibroblasts to promote kidney fibrosis through miR-150-containing exosomes. Exp Cell Res. 2020 Jul 15;392(2):112007.

86 Huang SJ, Huang J, Yan YB, Qiu J, Tan RQ, Liu Y, et al. The renoprotective effect of curcumin against cisplatin-induced acute kidney injury in mice: involvement of miR-181a/ PTEN axis. Ren Fail. 2020 Nov;42(1):350-7.

87 Lv W, Fan F, Wang Y, Gonzalez-Fernandez E, Wang C, Yang L, et al. Therapeutic potential of microRNAs for the treatment of renal fibrosis and CKD. Physiol Genomics. 2018 Jan 1;50(1):20-34.

88 Wang X, Xue N, Zhao S, Shi Y, Ding X, Fang Y. Upregulation of miR-382 contributes to renal fibrosis secondary to aristolochic acid-induced kidney injury via PTEN signaling pathway. Cell Death Dis. 2020 Aug 14;11(8):620. 